\title{
Application of Impulse Response Method in Identifying the Causes of Gold Price Fluctuation
}

\author{
Qing Cheng ${ }^{1}$, Jinpu $\mathrm{Jiao}^{2}$, Honghua Chen ${ }^{1}$, Fen $\mathrm{Xu}^{3 *}$ \\ ${ }^{1}$ College of Economics and Management, China Agricultural University, Beijing 100083, China \\ ${ }^{2}$ Shanghai Gold Exchange, Shanghai 200001, China \\ ${ }^{3}$ Tsinghua University, Beijing 100083, China
}

Corresponding Author Email: xufxinge@ 163.com

https://doi.org/10.18280/isi.240109

Received: 15 December 2018

Accepted: 10 January 2019

\section{Keywords:}

impulse response (IR), gold price

fluctuation, demand shock

\begin{abstract}
In the case of random signal interference, this paper uses the classical signal identification method of impulse response (IR) method to design the program for identifying the fluctuation of gold price and then obtain the identification model of gold price fluctuation, In this design, the gold price sequence, the gold manufacturing demand sequence and the gold inventory demand sequence were regarded as input signals to discuss the fluctuation amplitude of the sequence, and analyse the motivation of the large fluctuation in the gold price from the perspective of manufacturing demand, inventory demand and specific prevention demand. The results showed that after the decoupling of the gold and dollar in 1975, the specific prevention demand shock of gold was the main reason for price fluctuations, and the demand for gold manufacturing had little impact on the fluctuation of gold prices. The correlation between specific demand and gold price in the fluctuation can effectively explain the phenomenon of "buy expectations, sell facts" and also prove the validity of the model.
\end{abstract}

\section{INTRODUCTION}

In the digital signal processing, the impulse response is the reaction of the system to the input unit impulse function. For linear systems without random noise, when the input signal is an impulse function, the response of the system output is called the impulse response function. With the continuous advancement of science and technology, people's demand for digital signal identification has been continuously improved, since the identification efficiency directly determines the accuracy of prediction. In finance, accurate identification of digital signals is the key to profitability. Besides, following the continuous development of quantitative trading, the signal identification technology, as an important theoretical basis for digital signal identification and parameter estimation in quantitative trading, has important significance for digital identification accuracy in finance. Therefore, the need for quantitative trading has led to the continuous development of digital signal identification technology in the economic field. IR analysis enables people to use digital signal identification technology instead of artificial subjective judgments, to accurately identify price fluctuations from a huge amount of historical data, and formulate the excess return strategies.

Traditional digital signal identification methods mainly include maximum likelihood method, least square method and correlation analysis method etc. In 1975, Carl Friedrich Gauss proposed the least squares method to find the best function matching of data by minimizing the square of the error. Due to the advantages of convenient implementation and simple principle, it has been widely used, and become a milestone in the estimation theory. Later, based on the least square method, scholars derived many complementary methods, such as auxiliary variable method, multi-step least squares method, augmented matrix method and generalized least squares method; these methods have their respective advantages in identification. However, these traditional digital signal identification methods generally have certain limitations. For nonlinear systems, they fail to make accurate estimation, and can only obtain local optimal solutions.

Today's digital signal identification methods have made some improvements, mainly including wavelet transform [1], genetic algorithm [2], neural network [3], fuzzy logic [4], and IR etc. Shamma and Athans [5] proposed variable parameter models whose parameters can be directly measured or calculated. Xu et al. [6] solved the problem of digital signal identification by weighting each partial model in cubic spline method. Yang [7] used the local signal identification method to solve the partial missing problem of the output data, and weighted the local model by exponential function, to obtain the interpolation of the output data and then solve the global model. Jin et al. [8] improved the accuracy of digital signal identification by combining with the expectation maximization algorithm. The methods mentioned above each have their own advantages, but there also exist various interference factors in the actual economic phenomenon, which shall de-stabilize the model and affect the estimation results. And for most economic phenomena, the above methods are too theoretical. Therefore, more attention should be paid to serve practical problems with the theories, in order to better use digital signal identification technology and solve economic problems. For discrete systems, the impulse response function is an infinite weight sequence; the output sequence of the system is the convolution of the input sequence and the weight sequence, which can better identify the signal transmission process. Furthermore, the interference factor is reasonably added to the model in a simple and easy way. Thus, the IR method can well fit the price fluctuation process. 
In this paper, the IR analysis was conducted to identify the causes of gold price fluctuations. Through MATLAB programming, the gold price sequence, the gold inventory demand sequence and the gold manufacturing demand sequence were used as input signals to make digital signal identification of the causes for digital price fluctuation. Besides, a vector autoregression (VAR) model with three endogenous variables was adopted, allowing variables to interact with each other, so as to study the impact of manufacturing demand, inventory demand and other demands on gold prices, and search reasons for the sharp fluctuations in gold prices.

\section{ALGORITHM DESCRIPTION}

All the information that can describe the system characteristics is recorded as an impulse response function. The system is fully and effectively impulsed by the input data $X_{t}$ at the input end, and the output data $Y_{t}$ of the system is collected at the output. Moreover, in the actual economic phenomenon, there exists noise interference. In this paper, the random term was recored as $\alpha_{t}$.

For a linear system with multiple inputs, the transfer function model is given as:

$y_{t}=\sum_{j=1}^{k} \frac{\Omega_{j}(\mathrm{~B}) \mathrm{B}^{b_{j}}}{E_{j}(\mathrm{~B})} X_{i j}+\frac{\theta(\mathrm{B})}{\phi(\mathrm{B})} \alpha_{t}$

where, $\Omega(B), E(B), \Theta(B)$ and $\Phi(B)$ are polynomials of the backward shift operator $(B)$, and the orders are $s, r, q$, and $p$, respectively; $b$ is the delay parameter and $\alpha_{\mathrm{t}}$ is the random term of the independent and normal distribution. Let:

$V(\mathrm{~B})=\frac{\Omega_{j}(\mathrm{~B}) \mathrm{B}^{b_{j}}}{E_{j}(\mathrm{~B})}=v_{0}+v_{1} B+v_{2} B^{2}+\ldots$

where, $V(B)$ is a rational function of $B$, so it is also a B-infinite higher-order polynomial, which can be written as the expression in formula (2), and the coefficient $\mathrm{v}_{\mathrm{j}}(j=1,2, \ldots)$ of $V(B)$ is called impulse response function. To simplify the input variables to a variable, substitute the formula (2) into (1), and derive the cross-correlation function $\rho_{\mathrm{xy}}$ of the input variable $X_{t}$ and the output variable $Y_{t}$.

$$
\rho_{\text {xy }}(\mathrm{k})=\frac{\sigma_{x}}{\sigma_{y}}\left[v_{0} \rho_{\mathrm{x}}(\mathrm{k})+v_{1} \rho_{\mathrm{x}}(\mathrm{k}-1)+\ldots+v_{k} \rho_{\mathrm{x}}(0)+\ldots\right], \quad k=0, \pm 1, \pm 2, \ldots
$$

When the input sequence $X_{t}$ is a white noise sequence, the remainder of the formula on the right side is all zero except for $v_{k} \rho_{x}(0)$, since the autocorrelation function of the white noise sequence is 0 . Then, formula 3 can be simplified as $\rho_{\mathrm{xy}}(\mathrm{k})=\frac{\sigma_{x}}{\sigma_{y}} \mathrm{v}_{k}$.

$$
\rho_{\alpha \beta}(\mathrm{k})=\frac{\sigma_{\alpha}}{\sigma_{\beta}} \mathrm{v}_{k}
$$

The input variable $X_{t}$ and the output variable $Y_{t}$ are prewhitened into sequence $\alpha_{t}$ and $\beta_{t}$ respectively, then the cross- correlation function was obtained in formula (4) above. After that, according to the characteristics of the cross-correlation function, the order $r$ and $s$ of the transfer function polynomial as well as the delay parameter $b$ were decided. For the noise identification, based on the autocorrelation and partial autocorrelation functions of the noise, the order $p, q$ of the noise ARMA model were determined.

The impulse response function $\mathrm{vj}$ is the response of the linear system to the unit pulse input signal with the initial condition equal to zero. If the duration $t$ of the input impulse signal is small enough, the response of the system is essentially the same as the unit impulse response. Figure 1(a) shows the response curve of the first-order system impulse input signal; Figure 1(b), if the duration of impulse input signal is small enough, such as $t_{1}<0.1 T$, the response of the system shall be essentially the same as the unit impulse response.

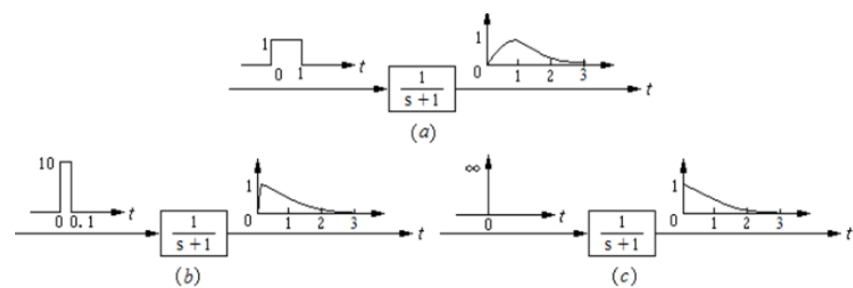

Figure 1. Unit impulse input of first-order system and its impulse curve

In Figure 2, the system input is an arbitrary function $r(t)$. If the input $r(t)$ can be approximated as multiple continuous impulse functions, the response of the impulse function can be derived separately, and then all response functions be superimposed by the superposition principle, to obtain the response of any input function.

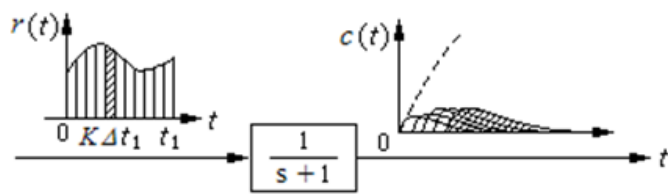

Figure 2. System response to any input function

\section{MODEL BUILDING}

\subsection{Demand shock on gold price fluctuations}

Many scholars have shown that gold price fluctuation will be affected by manufacturing demand, inventory demand and specific prevention demand. The Gold Yearbook pointed out that manufacturing demand includes gold demands for jewellery, electronics industry, dentistry, medical care; inventory demand includes gold coins, gold bars and gold medals, where demand for gold bars accounts for about $65.2 \%$ of inventory demand, and gold stock bars are mainly for exchanges traded funds and futures positions; specific prevention demands are the increased needs for gold by investors in case of political and economic turmoil etc. But it's also indicated in some documents that gold demand will be affected by gold prices. Therefore, the gold prices and different demands are no longer in a one-way causal relationship, because of the endogeneity between variables. Relatively speaking, the model allowing for endogeneity 
between variables can ensure more accurate results. Thus, the IR was applied in this paper to analyse the causes of gold price fluctuations. Based on the constructed VAR model containing three endogenous variables, an IR analysis was conducted to study the effects of gold manufacturing demand, gold inventory demand and specific prevention demand on gold price fluctuations, and to find out the reasons for the sharp fluctuations in gold prices.

In the VAR model, $\varepsilon$ represents a structural innovation vector that is continuously independent of each other.

$$
A_{0} Z_{t}=\alpha+\sum_{i=1}^{24} A_{i} Z_{t-i}+e_{t}
$$

In formula (5), it's assumed that $\mathrm{A}_{0}$ has a recursive structure, so that the error $e$ can be decomposed. The decomposition of $e$ is shown in formula (6). It is assumed in formula (6) that the gold manufacturing demand shock will not respond to the innovation of gold inventory demand in the same month. In practice, considering the cost of gold production adjustment and the uncertainty of the gold market situation, the response of manufacturing demand to inventory shocks is actually slow. Therefore, only the continuous increase in inventory demand is likely to increase manufacturing demand.

$$
e_{t} \equiv\left(\begin{array}{c}
e_{t}^{\text {manu }} \\
e_{t}^{\text {inven }} \\
e_{t}^{\text {spec }}
\end{array}\right)=\left[\begin{array}{ccc}
a_{11} & 0 & 0 \\
a_{21} & a_{22} & 0 \\
a_{31} & a_{32} & a_{33}
\end{array}\right]\left(\begin{array}{c}
\varepsilon_{t}^{\text {manu }} \\
\varepsilon_{t}^{\text {inven }} \\
\varepsilon_{t}^{\text {spec }}
\end{array}\right)
$$

Formula (6) means that the specific prevention demand shock of gold cannot be explained based on the innovation of the stock, which is equivalent to the mandatory exclusion limit, that is, the increase in the actual price of gold driven by the specific prevention demand innovation of the gold market will not immediately reduce the demand for gold stocks. But it will be delayed for at least a month. This limit is consistent with the weak performance of inventory demand after each major gold price increase in the sample.

In addition, the price of gold is expected to generate gold demand. Thus, if the price of gold is expected to rise, gold will be bought, and then sold after the price rises, thereby earning income; if the price is expected to fall, gold will be sold. This paper defines the gold demand arising from the lagged variable in the gold price as a specific prevention demand.

\subsection{Data sources}

This paper uses the monthly data from January 1975 to December 2016. The global gold inventory demand (INVD) data comes from the monthly average position data of the New York Mercantile Exchange that downloads from Wind, Levin and Wright (2006) found that the US Commodity Exchange's gold holdings had the greatest impact on gold prices, and also uses the intraday data over a 17-year period to find that market structure, market liquidity, daytime and macroeconomic announcements led to a faster response of US Commodity Exchange's futures position information to the price of gold Worthington \& Pahlavani, 2007 divided the daily transaction into three trading hours and found that the US commodity exchange's dominance of gold prices was significant across all trading hours. Gold price fluctuation data (PRICE) is the London Gold PM Fix Price downloaded from the World Gold
Council website. Using the US CPI reduction method proposed by Barssk and Kilian [9], the actual gold price was calculated based on the January 1975 dollar level, and then the continuous compound excess return rate was also calculated, that is, to subtract it in previous period after taking the natural logarithm. Global gold manufacturing demand (MAND) is the global shipping index downloaded from the Thomson Reuters database. There is no monthly data on global gold manufacturing demand. Thus, Pierdzioch et al. [10]; Baur and Lucey [11]; Baur and McDermott [12] point out that gold manufacturing demand is closely related to international business cycle indicators. Lutz Kilian [13] suggests the use of the global shipping index in price fluctuation which is an effective measure of the international cycle for the following reasons: First, the commonly used exchange rate-weighted average global industrial production index cannot measure each country's contribution to the economic activities of global entity, while the global shipping index can reduce the impact of financial variables, and reflect the global economic activities more objectively. Second, Asian gold consumption demand accounts for more than $60 \%$ of the world's gold consumption demand, as the main consumer market for gold. However, it's difficult for the global industrial production index to reflect the increase in the weight of Asian countries. The global shipping index can be free from exchange rate weighting, well reflecting Asia's economic weight. Third, Lutz Kilian indicated that the global shipping index is consistent with the important milestone facts, and that its main expansion and contraction time as well as their magnitude are all consistent with the nodes of important facts. This paper uses the global shipping index, which was built on January 1968 on a monthly basis.

\subsection{Software platform}

MATLAB is a combination of the first three letters of Matrix and Laboratory. It is a mathematical software tool issued by MathWorks in the United States. It can perform data interaction, numerical analysis and calculation, image generation and processing, etc., mainly including two parts: MATLAB and Simulink. This paper uses MATLAB programming to generate software modules for the identification of gold price fluctuations.

\section{EMPIRICAL ANALYSIS FOR THE CAUSES OF GOLD PRICE FLUCTUATIONS}

This paper uses IR to analyse the impact of manufacturing demand, inventory demand, and specific prevention demand on gold price fluctuation. Firstly, in order to ensure the stability of the data results, the unit root test was carried out. Secondly, the hysteresis test was performed to determine the lag order. Afterwards, the VAR model was constructed to analyse the impulse response function. Finally, the variance decomposition was performed.

\subsection{Model test}

Firstly, unit root test was carried out. This paper mainly lists the results of ADF test (Figure 3), which are consistent with the test results of other methods. All feature roots are in the unit circle without any unit root, indicating that the data is stable and can be analysed using the model. 
Then, the lag order was determined. The $3 \mathrm{D}$ vector autoregressive (VAR) model was constructed by using variables PRICE, INVD, and MAND. The lag order of the VAR model was determined by the lag length criterion in the lag structure. Table 1 lists the results. Four of the test indicators determine that the lag phase 7 is the optimal lag period. Therefore, a VAR model with a lag phase of 7 was established in this paper, i.e., VAR (7).

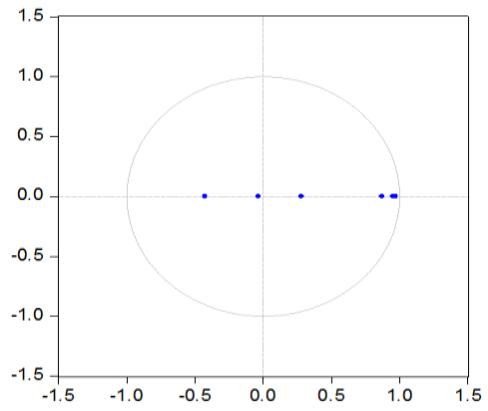

Figure 3. Inverse roots of AR characteristic polynomial

Table 1. Determining the lag order of VAR model

\begin{tabular}{|c|c|c|c|c|c|c|}
\hline Lag & LogL & LR & FPE & AIC & SC & HQ \\
\hline 0 & -10324.76 & NA & $5.36 \mathrm{e}+18$ & 51.63882 & 51.66875 & 51.65067 \\
\hline 1 & -9021.339 & 2580.780 & $8.28 \mathrm{e}+15$ & 45.16669 & 45.28644 & 45.21412 \\
\hline 2 & -8971.108 & 98.70433 & $6.74 \mathrm{e}+15$ & 44.96054 & $45.17009^{*}$ & 45.04352 \\
\hline 3 & -8961.575 & 18.58917 & $6.72 \mathrm{e}+15$ & 44.95787 & 45.25723 & 45.07641 \\
\hline 4 & -8923.123 & 74.40352 & $5.80 \mathrm{e}+15$ & 44.81062 & 45.19979 & $44.96473^{*}$ \\
\hline 5 & -8910.962 & 23.34989 & $5.71 \mathrm{e}+15$ & 44.79481 & 45.27379 & 44.98449 \\
\hline 6 & -8902.133 & 16.81902 & $5.72 \mathrm{e}+15$ & 44.79567 & 45.36445 & 45.02091 \\
\hline 7 & -8886.930 & $28.73449 *$ & $5.54 \mathrm{e}+15$ & $44.76465^{*}$ & 45.42324 & 45.02546 \\
\hline 8 & -8884.174 & 5.167880 & $5.72 \mathrm{e}+15$ & 44.79587 & 45.54427 & 45.09224 \\
\hline
\end{tabular}

\subsection{Identifying three structural shocks}

In this paper, the VAR model was used to break down the actual gold price fluctuations since 1975 into three shocks: the shock of demand in the gold inventory market, the shock of total demand in the gold manufacturing industry, and the shock of specific prevention demand (Figure 4).
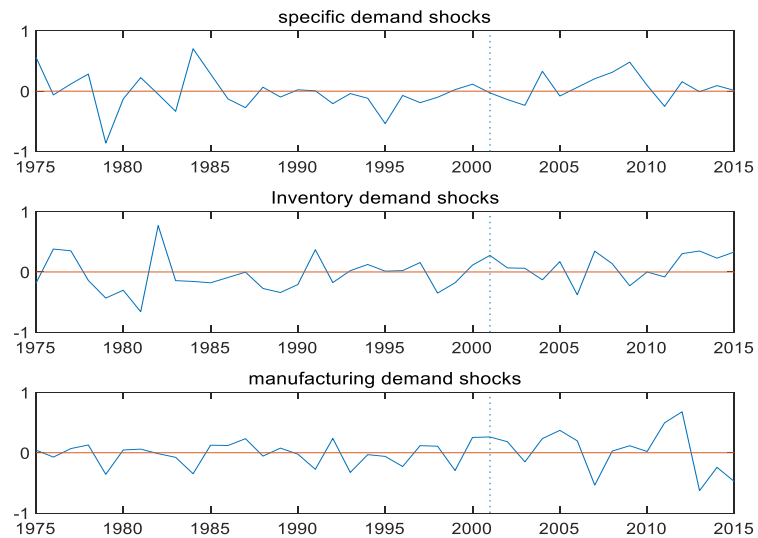

Figure 4. Shock decomposition of global gold inventory demand, manufacturing demand and specific prevention demand on real price fluctuations of gold

Since the price of gold is expected to generate gold demand, this paper defines the demand for gold due to the lag in the price of gold as specific demand. It can be seen that, in contrast, the shock of the gold manufacturing industry is not obvious, while that of inventory demand in the gold inventory market. Since 2000, the latest increase in the actual price of gold can be attributed to specific prevention demand shocks, especially, after the September 11th incident in the United States in 2001, the rise in gold prices was mainly due to the increase in demand for specific prevention demand. Through the analysis above, it's found that gold manufacturing demand, gold inventory demand and gold specific prevention demand have different impacts on gold price fluctuations, and these three kinds of demand changes are the demand drivers of gold price fluctuations.

\section{3 price \\ The impact of three structural shocks on real gold}

Figure 5 shows the use of IR to identify global gold inventory demand shocks, manufacturing demand shocks, and the impact of specific prevention demand shocks on gold price fluctuations. All shocks have been standardized.

The specific prevention demand for gold is negatively correlated with the price of gold. In the curve 3.3 of Figure 5 above, the specific prevention demand shock of gold led to an immediate sharp rise in global gold prices. Then, as expectations were adapted, the gains disappeared, and the high demand for gold would intensify the decline in gold prices, which was consistent with the rapid rise and fall of gold caused by market-specific prevention demand. The correlation between specific demand and gold price in the fluctuation can effectively explain the phenomenon of "buy expectations, sell facts" and also prove the validity of the model. Before the outbreak of political and economic events, the price of gold increased (decreased). However, when the expectation is adapted, and the fact comes into being, the price of gold would fall (rise). For instance, in the expectation of Fed's interest rate hike, the spot gold price continued to fall, whereas, after the announcement of the interest rate hike, the low gold price rebounded as expected. For sudden political events, gold prices are expected to rise rapidly, driven by a large number of safe-haven demand. But once the political situation is stable, the price of gold will immediately return to its original state. In the British general election, since the market didn't want Theresa to win, the risk aversion increased, and the gold price was expected to rise; but in the end Teresa won, plus other factors of the election night, the gold fell. Similarly, in the Dutch and French general election, the price of gold also fluctuates in the same way. 

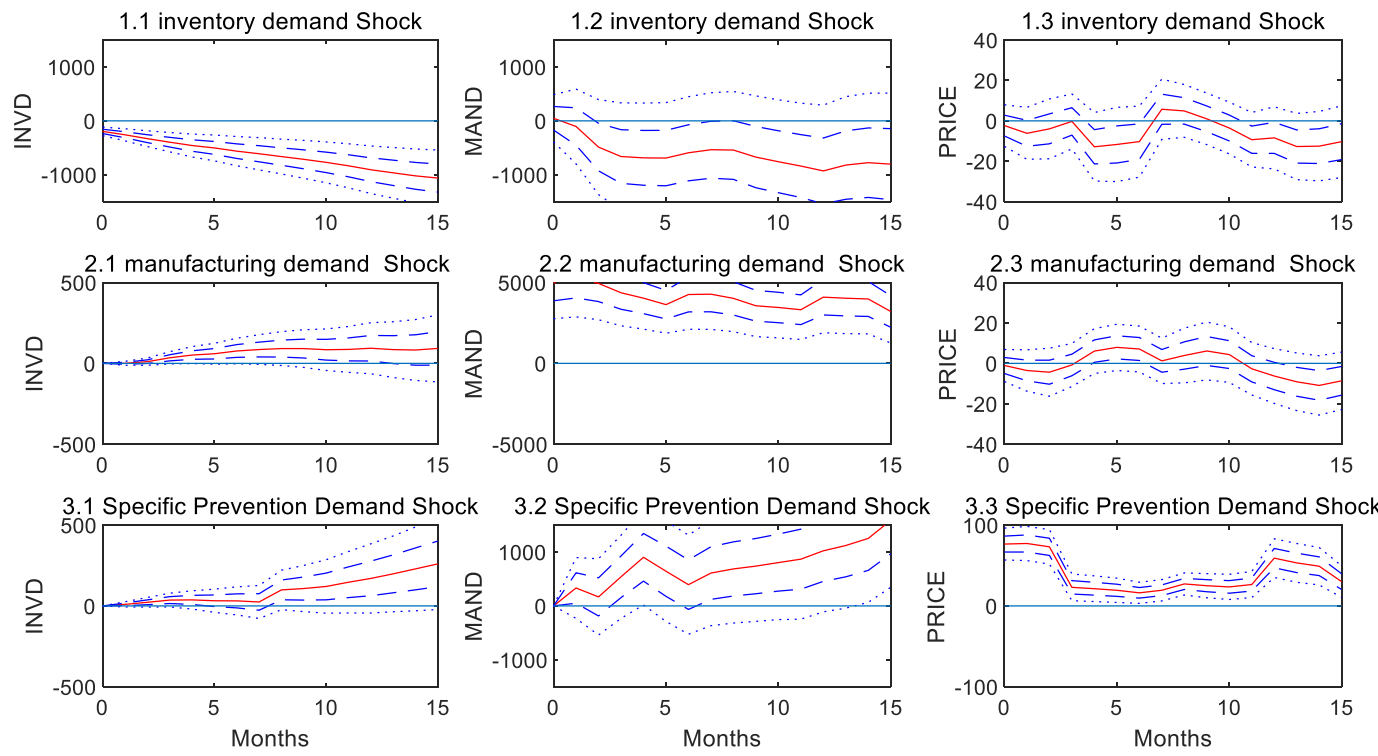

Figure 5. The impulse response of global gold inventory demand, manufacturing demand and specific prevention demand to real price of gold

Gold manufacturing demand has a weak impact on gold price fluctuation. In the curve 2.3 of Figure 5, the shock of gold manufacturing demand will not immediately lead to fluctuations in gold prices. The curve 1.3 of Figure 5 shows that the inventory demand shock can cause the global gold price to fluctuate slightly, and it has a greater impact on the gold price than the manufacturing demand, but the impact of the gold inventory demand on the actual price of gold is limited. Besides, after the political events, why could the actual price of gold rise sharply? For this question, it is due to an increase in specific prevention demands, as shown in 3.3 curve of Figure 5. A specific prevention demand shock will cause the sharp rise of gold price immediately and last for a long time.

In short, the shock of gold specific prevention demand will lead to a sharp rise in the price of gold, and the specific prevention demand is the main cause of fluctuations in gold prices. Gold manufacturing demand and inventory demand have limited impact on gold prices. But compared with gold inventory demand, gold manufacturing demand has a weaker impact.

\subsection{Cumulative impacts of three structural shocks on the real price of gold}

Figure 6 depicts the cumulative effect of each structural shock on the real price of gold since 1975 based on historical data decomposition. The first group showed that historically, the effect of gold manufacturing demand shocks on the real price fluctuation of gold was relatively small, and the biggest effect so far is driven by specific prevention demand in the gold market.

In summary, by quantifying the long-term and short-term cumulative effects of different shocks on gold prices, it's found that specific prevention demand is the main driver of gold price fluctuation, and the impact of inventory demand on gold price fluctuation is limited. Through studying the timing of specific prevention demand shocks in the gold market, it's further found that specific prevention demand shocks are related to consumers' preventive demands. Prevention demand is a consumer's preventive purchase demand to prevent uncertain events. It is related to the market's assessment of the turmoil in the future political and economic situation. The Preventive demand of gold is mainly to prevent the political and economic instability of the world. For instance, the real price increase of gold in 1975 was related to a sharp increase in specific prevention demand, while the effect of gold inventory demand shocks was small. After the Iranian revolution in late 1979 and 1980, continued concerns about the normal order of the world led to a huge increase in specific prevention demands, and the real price of gold rose rapidly. After the US airstrikes in Libya in 1986, these concerns further intensified, the preventive demand reached its peak, and the price of gold also reached its peak. Subsequently, the political economy tended to stabilize, and the cumulative effect of specific prevention demand in the gold market reached its peak valley in 1996, and the real price of gold also approached the bottom. Later, in 1997, with the outbreak of the Asian financial crisis, the Internet bubble burst; the 9/11 incident occurred, the demand for prevention continued to increase, and the price of gold continued to rise. In particular, the rise in gold prices after 2005 is entirely due to a cumulative effect by the positive shock of global specific prevention demand. Thus, the increased specific prevention demand driven by preventive demand has had a significant impact on gold price fluctuation.
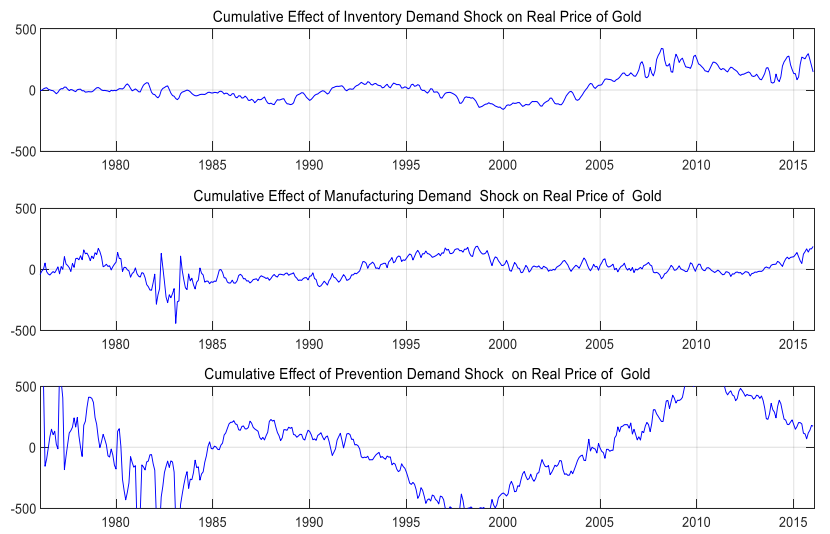

Figure 6. Cumulative effect of different price shocks on gold prices 


\section{CONCLUSIONS RECOMMENDATIONS}

AND

POLICY

In this paper, the signal identification of IR method was used to identify the causes of fluctuations in gold prices. It uses MATLAB as a platform to generate recognition programs. In today's economic phenomenon, the characteristics of price fluctuations are more complex and endogenous. The use of IR method to analyse the causes of gold price fluctuations can solve the endogeneity and random interference problems between economic variables. With the development of quantitative trading, a procedure for automatically identifying the characteristics of price fluctuations is quite necessary.

This paper uses the monthly data from January 1975 to December 2016. Based on the endogeneity between the three demands, the VAR model was applied to perform technical analysis and then both impulse response function and variance decomposition were used for further analysis. From the perspective of demand, the three drivers of gold price fluctuation were discussed: manufacturing demand, inventory demand and specific prevention demand. It's found that after the decoupling of the gold and dollar in 1975, the main reason for the fluctuation of gold price was the specific prevention demand shock, rather than the generally considered gold inventory demand shock. In addition, the specific prevention demand in the fluctuation of gold price is inversely proportional to the price of gold. These findings can effectively explain the phenomenon of "buy expectations, sell facts" and prove the validity of this model to explain realistic economic problems.

Based on the above conclusions, the recommendations have been made as follows: First, when consumers make gold consumption, they should not only pay attention to the price of gold but also the amount of change in the price of gold within a certain period of time. Second, when consumers make basic judgments and predict price changes through supply and demand, they must also focus on the impact of gold specific prevention demand on gold prices, and fully grasp the changes in specific prevention demand shocks in order to better predict gold prices. Third, the sources of shock possibly affecting the price of gold must be understood. In case of regional wars outburst, political turmoil or economic crisis, the specific prevention demand for gold will increase significantly, leading to a sharp increase in the price of gold within a short period of time. "Gold in troubled times" means not only that the gold is the hard currency, but also that during the turbulent period, the specific prevention demand of gold will lead to an increase in the price of gold and increase the return on investment portfolio. Accurate analysis for the source of gold price shocks is the key to obtaining excess returns.

\section{REFERENCES}

[1] Sureshbabu N, Farrell JA. (1999). Wavelet based system identification for nonlinear control. IEEE Transactions on Automatic Control 44(2): 412-417. https://doi.org/10.1109/9.746278

[2] Kristinsson K, Dumont GA. (1992). System identification and control using genetic algorithms. IEEE Transactions on Systems, Man and Cybernetics 22(5): 1034-1046. https://doi.org/10.1109/21.179842

[3] Xu XR, Pan ZQ, Li XH, Li JZ. (2019). Continuous hopfield neural network based on wavelet transform and overlapped peak resolution strategy. Journal of Dalian University of Technology (01): 97-105.

[4] Yang JW, He ZY. (2012). Study on recognition of fault transients using hybrid fuzzy petri net. Power System Technology 36(2): 250-256.

[5] Shamma JS, Athans M. (1991). Guaranteed properties of gain scheduled control for linear parameter-varying plants. Automatica 27(3): 559-564. https://doi.org/10.1016/0005-1098(91)90116-J

[6] Xu ZH, Zhao J, Qian JX. (2009). Nonlinear MPC using an identified LPV model. Industrial \& Engineering Chemistry Research 48(6): 3044-3051. http://dx.doi.org/10.1021/ie801057q

[7] Yang XQ. (2014). Linear parameters system identification method. Harbin Institute of Technology.

[8] Jin X, Huang B, Shook DS. (2011). Multiple model LPV approach to nonlinear process identification with EM algorithm. Journal of Process Control 21(1): 182-193. https://doi.org/10.1016/j.jprocont.2010.11.008

[9] Barsky RB, Kilian L. (2004). Oil and the macroeconomy since the 1970s. Journal of Economic Perspectives 18(4): 115-134. https://doi.org/10.1257/0895330042632708

[10] Pierdzioch C, Risse M, Rohloff S. (2014). On the efficiency of the gold market: Results of a real-time forecasting approach. International Review of Financial Analysis 32(3): 95-108. https://doi.org/10.1016/j.irfa.2014.01.012

[11] Baur DG, Lucey BM. (2010). Is gold a hedge or a safe haven? An analysis of stocks, bonds and gold. Financial Review 45(2): 217-229. http://dx.doi.org/10.1111/j.1540-6288.2010.00244.x

[12] Baur DG, Mcdermott TK. (2010). Is gold a safe haven? International evidence. Journal of Banking \& Finance 34(8):

1886-1898. https://doi.org/10.1016/j.jbankfin.2009.12.008

[13] Kilian L, Rebucci A, Spatafora N. (2007). Oil shocks and external balances. Journal of International Economics 77(2): 181-194. https://doi.org/10.1016/j.jinteco.2009.01.001 